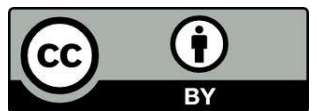

\title{
PAPEL DE LA RELIGIÓN EN LA SOCIEDAD DEL RIESGO
}

\author{
Role of religion in Society of risk
}

David Vides

Universidad La Salle de Bogotá

Doutorando em Educação

\begin{abstract}
RESUMEN: En el reconocimiento de la situación del ser humano en la actualidad, la globalización parece constituirse en un punto de partida fundamental; ahora, la universalización y homogeneización no se han dado únicamente en escenarios sociales y económicos sino que atraviesan factores como el imaginario, la sensibilidad y las actitudes. En consecuencia, la humanidad se enfrenta a la modificación de dinámicas sociales, políticas, económicas y culturales, que terminan afectando a las personas concretas en el campo de las subjetividades, las cosmovisiones y los estilos de vida. En ese escenario, el artículo plantea la religión como posible puerta de entrada para la comprensión de la situación del hombre en el mundo posterior al moderno; para ello, luego de algunas consideraciones iniciales, expone elementos de la Sociedad del Riesgo que encuentran eco y cabida en el campo de lo religioso, siguiendo los planteamientos de Josetxo Beriain, Anthony Giddens y Zigmunt Bauman. Finaliza, exponiendo algunas implicaciones que conlleva la consideración de la dimensión trascendente del hombre en el ámbito político, y, las relaciones con el proyecto de investigación La Educación Religiosa Escolar como espacio de formación política.
\end{abstract}

Palabras clave: Religión, Riesgo, Modernidad, Trascendencia, Política.

ABSTRACT: In recognition of the situation of human beings today, globalization seems to become a fundamental starting point; Now, globalization and homogenization is not only have social and economic scenarios but factors such as the imaginary cross, sensitivity and attitudes. Consequently, mankind is facing changing social, political, economic and cultural dynamics, which end up affecting real people in the field of subjectivities, world views and lifestyles. In that scenario, the article discusses religion as a possible gateway to the understanding of the human situation in the post-modern world; for that, after some initial considerations, it exposes elements of society that are echoed Risk and place in the field of religion, following the approach of Josetxo Beriain, Anthony Giddens and Zygmunt Bauman. Ends, exposing some implications of the consideration of the transcendent dimension of man in politics, and relations with the research project School Religious Education as a space for political education..

Keywords: Religion, Risk, Modernity Transcendence Policy. 


\section{Introducción}

Una consideración de las bases de la modernidad permite vincular la situación del hombre contemporáneo a un problema de sentido coherente con algunos elementos tradicionalmente relacionados con la dimensión trascendente. Elementos como la necesidad de un abordaje holista de la realidad, las implicaciones de la conciencia moderna en la vida individual, y, las nuevas comprensiones de la alteridad, remiten a la consideración del papel de lo religioso en la Sociedad del Riesgo (Beriain, 1996); de hecho, si "pensar en términos de riesgo es vital para evaluar la divergencia entre los proyectos preconcebidos y sus resultados consumados" (Giddens en Beriain, p. 36), el recorrido de la religión puede ser una muestra clara de ello: el afán por la consecución de una sociedad positivista y racional, donde lo especulativo y lo religioso no tenían cabida, resultó en la producción del mito de lo indeterminado, y a su vez incalculable, esto es, fuera del alcance del dominio racional; "parafraseando a Adorno y a Horkheimer en su Dialéctica de la Ilustración podemos decir que el riesgo como secularización de la fortuna de las sociedades tradicionales revierte en mitología, ya que su incalculabilidad es indeterminada" (p. 23). Asimismo, fenómenos como la sociedad de consumo, mientras recrudecen el neoliberalismo o neocapitalismo, dan muestra a nivel antropológico de un hedonismo extremo y un materialismo, que para Beuchot (2010) llega a extremos nihilistas "en el sentido de decadentismo, de oposición a lo establecido, sin ningún propósito de cambio, sin ninguna idea clara de lo que se va a hacer o lograr" (p. 35).

Sin embargo, pese a la idea de resquebrajamiento de los metarrelatos y los escenarios totalizantes de la modernidad, en el tránsito a la sociedad del riesgo se mantiene la preocupación por la existencia de un componente que dé unidad a la humanidad como especie y como construcción cultural. De tal manera, se presenta la religión como alternativa holística y de unidad frente a la fragmentación, que puede contribuir a una mejor comprensión de la situación del hombre en el mundo contemporáneo. En este sentido, cabe decir que se asume una comprensión de lo religioso desde Raimon Panikkar (2007) como mediación para un objetivo de plenitud en el ser humano; esta consideración integra en una sola dinámica elementos opuestos desde las consideraciones dualistas.

Dos ideas nos permiten establecer vínculos iniciales de la Modernidad tardía 
contemporánea con el ámbito de lo religioso: en primer lugar, la certeza de que "la modernidad altera radicalmente la naturaleza de la vida cotidiana y afecta a las dimensiones más íntimas de nuestra experiencia" (Giddens en Beriain, p. 33), evidencia que el establecimiento de un estado Moderno no sólo es asunto del dinamismo e impacto global de las instituciones sino que ejerce importante influencia en las convicciones, el comportamiento y la vida social de las personas, ejerciendo un influjo oscilante entre "influencias globalizantes por un lado y disposiciones personales por el otro" (p. 34). En segundo lugar, el surgimiento de una conexión en la humanidad, más allá de la hipercomunicación contemporánea; esto es, un vínculo en los sentires, que se expresa en movimientos mundiales, y que para Beriain, se relaciona con una sensación de riesgo y peligro:

El peligro nos convierte a todos en vecinos de Chernobyl, en ciudadanos de Ucrania, y lo mismo cabe decir con el "agujero de ozono" y el "efecto invernadero". Vivimos al lado del pulmón amazónico y de los casquetes polares. El discurso de la angustia-miedo que surge en la sociedad civil hoy frente a las amenazas económicas, ecológicas y militares, es un sustituto de las cosmovisiones holistas, en medio de la diferenciación funcional. La angustiamiedo no puede ser banida por los sistemas políticos, económicos o militares, es auténtica e inmune a la refutación (1996, p. 26)

Con estos presupuestos adquieren importancia los planteamientos de algunos autores que establecen conexión entre la sociedad del riesgo y lo religioso. A continuación, se plantean: la idea de contingencia siguiendo a Josetxo Beriain, un diálogo entre la seguridad ontológica de Giddens y la Fe como confianza en Panikkar, algunas ideas sobre la eternidad y la finitud en Zigmunt Bauman, y finalmente, algunas posibilidades para la comprensión del papel de la religión en el mundo moderno, con énfasis en las implicaciones en el ámbito político.

\section{La idea de contingencia en relación con la religión en el mundo moderno}

En el prólogo de Las consecuencias perversas de la modernidad. Modernidad, Contingencia y Riesgo (1996), Josetxo Beriain plantea que la función tradicional de la religión, ante un mundo necesariamente contingente, es la búsqueda de un último 
significado o sentido a "los significados contingentes, paradójicos o contradictorios que se derivan de la experiencia del hombre" (p.19). Para ello, ha utilizado fórmulas como Dios o el Karma en un intento de explicación de "por qué las cosas tienen que suceder, la forma en que lo hacen y que siempre pudieran ser diferentes"; de allí, Beriain deduce la elaboración de significados como un proceso fundamental en la formación de cualquier sociedad en cuanto éstos "introducen orden dentro de un caos (natural) potencialmente infinito"; en últimas, "la religión busca la «transformación de lo indeterminado en determinado»" (p. 19).

En este sentido, la contingencia queda siempre remitida a una "necesidad supramodal" que se expresaría en la fórmula Dios, como ente observador, generador y observador de todo, incluso la futura contingencia; en otras palabras, se establece una generalización dogmática en torno a Dios, cuyo atributo principal es la perfección, negación de la contingencia: "Toda la contingencia de un mundo crecientemente complejo, incluyendo el mal y la posibilidad de superarlo, debe ser atribuida a un Dios, y debe, por tanto, ser interpretada dentro del sistema religioso" (p. 19-20). En este sistema la dualidad se troca en una posibilidad de transformación de un elemento en otro -del pecado a la gracia, por ejemplo-, mediante una interacción social determinada -la penitencia, a partir del mismo ejemplo-: "El sufrimiento y la gracia reciben su necesidad de la decisión divina o del destino y reciben su contingencia de la relación (social) dentro de la dualidad. (p. 20).

Ahora bien, la ruptura de este esquema tradicional, al desplazar la religión al ámbito de lo privado, ha despojado al sistema social de la fórmula reductora de la contingencia (Dios), sirviéndose de una observación de segundo orden, ya no totalizante sino fragmentada. Se establece entonces, la necesidad de una "transformación de las estructuras simbólicas directivas de comunicación porque de otra forma los nuevos niveles requeridos de combinación de expectativas y rendimientos estructuralmente discrepantes no pueden ser reproducidos de forma operativa" (p.20). De esta manera, aparecen los códigos binarios como construcciones totalizadoras del mundo "con exigencias de universalidad y sin limitación ontológica", esto es, que ante la ausencia de una tercera posibilidad, todo lo que abarca su campo de comprensión -que es todo-, se subordinará obligatoriamente a uno u otro valor del código mismo; la consecuencia directa de esta totalización es "una contingencia sin excepciones de todos 
los fenómenos", en donde sin más alternativas se omiten la diversidad y los matices operando una reducción a lo positivo/negativo: "Estos códigos despliegan distinciones directrices como «tener/no tener» en la economía, «gobierno/oposición» en la política, «autenticidad/no autenticidad» en la cultura y el arte, «verdad/falsedad» en la ciencia, «justo/injusto» en el derecho, etc.” (p. 21). Teniendo en cuenta, la fragmentación producida por la diferenciación funcional, cada sistema busca dar alcance a su función en uno de los polos de la dualidad propuesta, sin embargo, encuentra un obstáculo significativo en

el incremento de contingencia (directamente proporcional al incremento de opciones) que se produce en las sociedades modernas por la inexistencia de una fórmula de reducción de contingencia del tipo «Dios», y por consiguiente por el distanciamiento entre la experiencia (pasado) y las expectativas (futuro), así como por la producción social creciente de ambivalencia introduciendo la posibilidad real de la alternativa entre la cooperación y el conflicto, entre el consenso y el disenso, etc. (p. 22).

De esta forma, se regresa a la paradoja de la sociedad del riesgo: a mayor determinación, en este caso, producida por la diferenciación funcional, mayor ambivalencia y posibilidades de opciones y riesgos. La comprensión racional del mundo suscita el surgimiento de un nuevo destino culturalmente producido: un retorno a lo mítico frente a un futuro indeterminado donde el destino no corresponde a fórmulas como la naturaleza o Dios, sino a la sociedad misma que se convierte en su nueva generadora.

Del paso de la fortuna medieval al riesgo moderno no se ha producido un nuevo mito social. Sencillamente se ha pasado del destino dado metasocialmente, desde una exterioridad metasocial, Dios, la naturaleza, al destino producido socialmente como consecuencia de la multiplicación de la franja de posibilidades de riesgo de altas consecuencias (p. 23).

En esta perspectiva, más allá del retorno a la fórmula reductora de contingencia, 
o bien, a la dicotomía invisibilizadora de la diferencia, un primer reto que se percibe en el horizonte de lo religioso es la confrontación de la conciencia moderna que en un intento vano de absolutizar la determinación, ahora se enfrenta al riesgo como un nuevo destino, desde los daños colaterales de una comprensión fragmentada de la realidad.

\section{La seguridad ontológica de Giddens y la fe como confianza de Panikkar}

La presentación de Anthony Giddens de las relaciones entre Modernidad y Autoidentidad, encuentra en la "seguridad ontológica" uno de sus puntos clave. Para Giddens, este término "hace referencia a la confianza que la mayor parte de los seres humanos tenemos en la continuidad de nuestra identidad y en la continuidad de nuestros entornos sociales y naturales de acción"; implica la experiencia que tiene el hombre de sí mismo "en relación a un mundo de personas y de objetos organizados simbólicamente, a través de la confianza básica" (en Beriain, 1996, p. 26). De tal forma, frente a mecanismos de deshumanización y sistemas abstractos que olvidan el ser personal concreto, la confianza adquiere importancia fundamental para el desarrollo de la personalidad y la potenciación de aspectos indispensables para el ser humano en plenitud. Se comprende entonces la confianza como defensa y protección frente a amenazas y peligros potenciales, evitando consecuencias en la voluntad o vivencias de abatimiento surgidas de acontecimientos peligrosos al ser considerados en toda su magnitud. Entra en juego aquí la consideración de la confianza como "medio de interacción con los sistemas abstractos que vacían a la vida cotidiana de su contenido tradicional y establecen influencias globales. Aquí la confianza genera un «salto hacia la fe» que exige compromisos prácticos" (p. 36).

De esta concepción de la seguridad ontológica, Giddens deriva consecuencias en el campo de lo que denomina "transformación de la intimidad", específicamente el surgimiento de las "relaciones puras" como prototípicas de las nuevas esferas de la vida personal. Por un lado, las relaciones puras subsisten en sí mismas por las satisfacciones que puedan proporcionar; esto es, se dejan de lado los criterios externos a la relación, y en consecuencia, la confianza no se suscita a partir de relaciones de deber, obligación o parentesco, sino únicamente por un proceso de apertura mutua (p. 39). Por otro lado, "las relaciones puras presuponen el «compromiso», que es una especie particular de confianza"; éste se entiende como un evento autoreferenciado: se da desde la exigencia de intimidad resultado del compromiso mismo con la relación y con las personas 
implicadas (p. 40).

Dicho lo anterior, si bien Giddens, no plantea elementos directos de conexión con lo religioso, más allá de la alusión al "salto de fe" (p. 36), aspectos como la transformación de la intimidad y sus implicaciones en el campo relacional, sí permiten el establecimiento de ciertas relaciones con las concepciones sobre la fe planteadas por Panikkar (2007). En principio, es necesario aclarar que Panikkar presenta la fe como una dimensión constitutiva del ser humano, evitando cualquier adhesión a una doctrina con tintes de exclusividad; esta inclusión de la Fe en lo constitutivo del hombre, supone su distinción de otros seres y por tanto es fundamento para la unión de la humanidad. Ahora bien, en concreto la Fe aparece como una dimensión que posibilita la perfección, de donde se derivaría su consideración como fundamento real de la existencia humana; de hecho, en esta perspectiva, el hombre estaría proyectado para funcionar desde la Fe, pues con ella surge la conciencia de que el fundamento del ser humano no está en sí mismo. No obstante, más que la creencia en un superior, el contenido de esta conciencia es la relación ontológica con lo absoluto estableciendo una ligación significativa con la realidad como un todo; es decir, la Fe libera al hombre de la simple existencia cósmica como dependencia del reino de los objetos.

La ruptura histórica de la modernidad ha jugado un papel importante en esta conciencia de la humanidad en cuanto tal; la superación de una conciencia fundada en la razón como forma de comunicación universal ha dado paso a la consideración de un escenario concreto y práctico en el cual la Fe, más que un supuesto especulativo abstracto, se convierte en una pregunta por lo que es posible ser. En otras palabras, se convierte en capacidad y deseo mediante el cual el hombre, llega a ser él mismo, es decir, logra alcanzar su plenitud. Si el contenido de esta apertura existencial es ser lo que aun no se es, evolucionar, avanzar hacia la plenitud, la Fe puede asumirse como necesidad, precariedad, -soporte de impotencia, insatisfacción y anhelo-, pero asimismo, posibilidad de superación, y en cuanto tal, se constituye en el fundamento de la dignidad humana, ya que remite a la comprensión del hombre como ser abierto e infinito, libre desde la toma de decisiones y la espontaneidad, y, creador de posibilidades (p. 227).

Dicho lo anterior, Giddens y Pannikar, plantean postulados coincidentes y complementarios en relación con fundamentos existenciales para el desarrollo de las potencialidades del ser humano. Las consideraciones sobre el establecimiento de una 
relación con el mundo, la primacía por un desarrollo auténtico del ser humano y el planteamiento de la apertura como eje fundamental en el ámbito relacional, se convierten en aspectos susceptibles de profundización, en el marco de una construcción antropológica que sustente la primacía de la dignidad de la persona fundada ya no en criterios objetivos sino en la persona concreta que se constituye en el máximo valor posible.

La experiencia de la libertad puede constituirse en expresión de esta complementariedad. Si ésta se comprende, más que una espontaneidad instintiva o una liberación política, implica lo que Panikkar (2007) denomina una "espontaneidad ontológica”, que permite al hombre hacerse a sí mismo y al mundo. Lo anterior sólo es posible en la independencia absoluta, imposible para el hombre por su historicidad, salvo cuando logra que su pasado sea "perdonado, destruido, transformado" para dar paso a un nuevo futuro; es el acto creativo, el único perfectamente libre: "sino crea no es libre, se limita a volver a mezclar las condiciones ya dadas" (p. 455). De tal manera, el ser humano sólo posee autonomía plena en un sector específico: él mismo, cuando forja su propio destino, de donde, la verdadera libertad se constituya en la posibilidad de llegar a ser él mismo en superación de los sistemas abstractos y las condiciones adversas, y en ello, la confianza y la fe cumplen un papel fundamental. Más aún, dice Panikkar: "El libre acto humano tiene en sí mismo la máxima dignidad humana posible, a la cual hay que subordinar cualquier otra cosa" (p. 460).

\section{La eternidad y la finitud en Zigmunt Bauman}

Con Bauman, aparece una aproximación a la religión como medio de comprensión del mundo contemporáneo; sus ideas sobre la eternidad y la finitud, tomadas de Vidas desperdiciadas. La modernidad y sus parias (2004) abren un horizonte complementario a los acercamientos sobre la contingencia y la confianza ya expuestos.

Para Bauman, la eternidad ha sido uno de los pocos universales genuinos, de hecho, "la conciencia de la eternidad (o más bien, deberíamos decir, la creencia en la eternidad) puede concebirse como uno de los rasgos definitorios de la humanidad" (p. 131). Ahora bien, la visión que se presenta de la infinitud es la de la incompatibilidad con la exclusión y la exención, esto es, en contra de la producción y destrucción de residuos humanos, "en la infinitud del tiempo y el espacio todo puede suceder y todo 
debe suceder" (p. 123). De tal forma, nada de lo que sucede puede ser considerado superfluo, innecesario o desechable, más aún "fuese lo que fuese, debe haber formado parte de un designio de Dios y de la Cadena Divina del Ser" (p. 124).

Lo anterior, conllevó en la modernidad, el contraste entre la brevedad de la existencia personal y la solidez del mundo que se consideraba eterno; con la licuefacción del mundo, entró en crisis igualmente, la idea de la infinitud: "Si la vida premoderna era una escenificación cotidiana de la infinita duración de todo excepto de la vida mortal, la líquida vida moderna es una escenificación cotidiana de la transitoriedad universal" (p. 126). Frente a la fugacidad de la vida individual, surge la cultura como mecanismo que en reconocimiento de la mortalidad "consigue redefinir de algún modo el horror ante la muerte como una fuerza motriz de la vida" (p. 126127); se basa en la esperanza que las creaciones y elaboraciones personales y sociales tienen un significado perdurable y trascenderán más allá de la muerte. En ese sentido, se puede afirmar que la cultura reemplazó la religiosidad tradicional en su tarea de conectar la vida mortal con la eternidad del mundo; en ello, el lenguaje cumple un papel fundamental en cuanto permite la desconexión de la inmediata realidad de la experiencia y la elaboración de mundos distintos a la realidad que nos contiene. El lenguaje nos permite la recreación del mundo cuando el ser individual esté ausente de él, de hecho, "en la invención de la eternidad radica, en efecto la magia del lenguaje" (p. 132); éste se convierte en vínculo entre la vida personal, destinada a terminar, y el mundo, destinado a permanecer para siempre. La relación de estos postulados con las funciones de la religión en el campo de la producción cultural y de sentido existencial, son múltiples.

En esa misma línea, el hecho de que la conciencia de la finitud y la muerte se liga con la eternidad, expresa la lógica inversamente proporcional a la que se hizo alusión en las consideraciones iniciales sobre la sociedad del riesgo; esto se ratifica más claramente en el hecho de que el lenguaje de expresión relacionado con la muerte y la eternidad admita alternativas de unión entre binomios tradicionalmente disociados, como el temor y la esperanza.

Para terminar este apartado, dos elementos finales derivados de Bauman en relación con la religión como referente de comprensión de la situación contemporánea. En primer lugar, el reconocimiento de que no es la religión como creencia per se sino el 
abordaje de la realidad y su contingencia, el que permite reconocer las consecuencias de un egoísmo desmedido y de una búsqueda exclusiva e individual de la felicidad; en este sentido, lo que hace falta es el componente político de búsqueda del bien común, reconocimiento del otro y conciencia comunitaria. En segundo lugar, una moción contextual en relación con cierta radicalidad del autor frente a lo efímero y lo líquido, cuando efectivamente, la religión alude a la unidad y a la existencia de elementos firmes a los cuales aferrarse; de hecho nuestra realidad latinoamericana en ciertos aspectos como la importancia simbólica de la tierra, y la autenticidad y solidez de las relaciones interpersonales, es muestra de una producción cultural que si bien permea las tendencias globalizantes, también refleja una aproximación a la trascendencia que se constituye en factor potenciador en el ámbito cultural y político.

\section{Posibilidades para el papel de la religión en la sociedad del riesgo}

Expuestas las ideas de Beriain, Giddens, Panikkar y Bauman, se hacen evidentes algunos elementos que vinculan la situación del hombre en la Sociedad del Riesgo, con el ámbito de lo trascendente y lo religioso. En ese sentido, valga la alusión a dos afirmaciones de Giddens: "La carencia de significado personal -el sentimiento de que la vida no tiene valor alguno que ofrecer- se convierte en un problema psíquico fundamental en el contexto de la modernidad tardía" y más adelante, "el «aislamiento existencial» es, no tanto una separación de los individuos entre sí, como una separación de los recursos morales necesarios para vivir en plenitud" (en Beriain, 1996, p. 42). Dado esto, se presentan algunas posibilidades para el ámbito de lo religioso como respuesta a la sensación frente a lo inesperado e inaprensible, el misterio.

Primero, la denuncia que hace Bauman sobre la autonomía territorial y funcional, que termina expresándose "en el derecho a no ampliar la mirada más allá del cerco y a no ser mirado más allá de él", esto es, "el derecho a decidir cuando se mantienen los ojos abiertos y cuando conviene cerrarlos" (en Beriain, 1996, p. 88). La fragmentación del mundo deriva en evasión de la responsabilidad personal y social, y el principio holístico de la realidad se reduce a una suerte de administrador que dirige una burocracia. Frente a este escenario de desmembramiento del mundo, la religión ha sido reducida a su propio cerco quedando separada de las intencionalidades que fluyen por el contingente social; el reto se plantea en términos de recuperación de una comprensión 
holística de lo social, donde las interconexiones permitan una reconfiguración de sentidos en las redes, imaginarios y estilos de vida de las personas concretas.

Segundo, el planteamiento de los foci imaginarii como horizontes de sentido. Dice el mismo Bauman: "Como todos los horizontes, nunca pueden alcanzarse. Como todos los horizontes, hacen posible el decurso de la vida con un propósito definido. (...) Confieren al itinerario la ilusión de destino, dirección y cometido" (p. 85). Ahora, en el cuestionamiento de lo religioso como un posible foci imaginarii, surgen dos opciones complementarias: alimentar la necesidad de un sentido para la existencia y vincularse con el misterio como supuesto fundamental de la dimensión trascendente. El reconocimiento de los ideales en su misión de "guiarnos hasta el territorio todavía inexplorado y no cartografiado" (Bauman, 2004, p. 148), haría parte fundamental de la experiencia humana de vivir más allá del mundo presente que experimentamos.

Tercero, los innombrables de Derrida, como aproximación a la comprensión de lo religioso en cuanto posibilidad alternativa en superación de las visiones dualistas. Los innombrables "no pueden ser incluidos dentro de la oposición (binaria) filosófica, a la que niega y desorganiza, sin constituir un tercer término sin dar salida a una solución bajo la forma de dialécticas especulativas" (Bauman en Beriain, p. 95); Al no ser lo uno ni lo otro, sino los dos al tiempo e incluso ninguno, se abre una puerta de entrada a lo inefable y al misterio, donde la ambivalencia y la indeterminación juegan a favor de una comprensión cercana al campo de lo religioso y lo trascendente.

Cuarto, ante un panorama de consumo y fugacidad, es imposible negar la existencia de "una desesperada búsqueda de raíces, parentesco, amistad y amor" (Bauman, 2004, p. 167). La necesidad auténtica por relaciones de intimidad en superación de la superficialidad de la modernidad tardía, termina siendo expresión de una aspiración más profunda por la infinitud y la eternidad, sin embargo, el compromiso permanente no parece ser una opción y empieza a desvanecerse la posibilidad de un destino compartido o un sentimiento de fraternidad. En ello, aparece un último reto, la recuperación de lo religioso en el sentido de lo auténticamente humano que pervive pese a las dinámicas pos modernas instauradas y denunciadas por los distintos autores.

Para terminar este apartado, cabe insistir en las conexiones entre elementos como el destino, las comprensiones religiosas e incluso la fórmula Dios, con los referentes de producción cultural, en las reflexiones hechas hasta el momento. Aparece 
entonces una veta de profundización desde las nuevas formas de manifestación de lo religioso en lo cultural, o bien, las nuevas manifestaciones religiosas producidas culturalmente. Sirvan de colofón, las palabras de Bauman:

Fue en los albores de la modernidad cuando se descubrió que la cultura se había estado ocultando tras el Dios que habla. Ahora le correspondía a la cultura, que habían hecho y seguían haciendo los hombres, hacerse cargo de la tarea de conectar la vida mortal con la eternidad del mundo y destilar una pizca de solidez y de duración a partir del impetuoso flujo de logros humanos pasajeros" (2004, p. 135)

\section{Implicaciones de la consideración de la dimensión trascendente en el ámbito político}

Teniendo en cuenta lo anterior, se abre paso la consideración de los elementos propios de lo religioso, que pueden incidir en el desempeño del individuo en el ámbito político. De hecho, el replanteamiento de los mecanismos reguladores de la vida social, donde empiezan a entrar en juego dinamismos subjetivos como la vergüenza y la culpa, abre una puerta para la consideración de nuevas formas políticas en relación con lo trascendente que asumen un nivel de compromiso "político-vital"; en palabras de Giddens, "una agenda política de la vida" que "exige un encuentro con específicos dilemas morales y nos obliga a plantear las cuestiones existenciales que la modernidad ha excluido institucionalmente" (En Beriain, 1996, p. 43). La consideración de las reformas en su relación con los manejos de poder y su influencia sobre las subjetividades van en esta misma línea. Dicho esto, la invitación es la de considerar los elementos que ligados con la trascendencia, pueden contribuir a la configuración de formas alternativas de ciudadanía y ejercicio político: "No hay que huir de él, del destino como hoy con tanto gusto se hace, sino que hay que seguir primero sus caminos hasta el fin para averiguar cuáles son sus poderes y sus límites" (Beriain, 1996, p. 17).

La fragmentación del mundo, a nivel cultural, y de la sociedad, a nivel funcional, exige entonces la atención a la diversidad que asume el reconocimiento del otro como un compromiso político vital y afecta la reflexión personal y comunitaria sobre cuestiones morales que adquieren cada vez mayor relevancia en el ámbito de lo público. La expresión más clara de este surgimiento es la aparición de nuevos movimientos 
sociales como dinamizadores de políticas emancipatorias en el marco de la pluralidad de opciones y estilos de vida propia de la sociedad del riesgo. Frente a ello, a continuación se presentan cuatro aspectos a tener en cuenta.

Primero, la consideración del tema de la verdad y su unión al poder. De hecho para Espinal (2006) "la verdad no se puede liberar de un sistema de poder, la verdad no está fuera del poder, la verdad es poder; entonces, la tarea consiste en aislar el poder de la verdad de las formas hegemónicas, de ahí que la cuestión política es la verdad misma"(p. 105). En la misma línea, Bauman retoma los planteamientos de Paul Ricoeur (1979) sobre la unión de la verdad con la violencia a partir de las instancias clerical y política, señalando que "la clerical no fue nada más que la esfera intelectual puesta al servicio de lo político o lo intelectual con ambiciones políticas", dicho lo cual, "el maridaje de la verdad y violencia es el significado de la esfera política" (En Beriain, 1996, p. 82 y 83). Con estos dos referentes, la necesidad de una aproximación al concepto de Verdad, se hace patente, más aún, en la línea de las humanidades y su comprensión holística del ser humano que en circunstancias como las actuales se convierten en "lugar por excelencia de disidencia, una especie de principio de desobediencia civil en nombre de una justicia de pensamiento" (Espinal, 2006, p. 98).

Segundo, la necesidad de una comprensión alternativa al tema de la tolerancia, "que muy frecuentemente significa: tú eres detestable, pero yo, siendo generoso, permitiré que sigas víviendo" (Bauman en Beriain, 1996, p. 82). Bauman ratifica esta idea con las palabras de Susan Mendus:

La tolerancia implica que el objeto tolerado es moralmente censurable. Hablar de tolerancia supone que es el descrédito que un sujeto mantiene con insistencia el que es objeto de tolerancia. La tolerancia no incluye la aceptación del valor del otro; por el contrario, es una vez más, tal vez de manera más sutil y subterránea, la forma de reafirmar la inferioridad del otro y sirve de antesala a la intención de acabar con su especificidad -junto a la invitación al otro a cooperar en la consumación de lo inevitable (p. 82).

Tercero, la aproximación al hombre como homo eligens, frente a una búsqueda de perfección inherente a sí mismo. En ese sentido, el inacabamiento del ser humano se 
constituye tanto en motivación como condena, y la apertura a un horizonte de posibilidades es al mismo tiempo la puerta de entrada del riesgo, con sus consecuentes sentimientos de miedo y frustración. De esta forma, es posible hablar con Bauman (2004), de la agonía de la elección, donde la figura del "Paraíso" se entiende como "esa amable y despreocupada condición que consiste en la no-necesidad de elegir y en la liberación de la premonición de que los actos pueden ser buenos o malos" (p. 135); aparece allí un imaginario religioso entendido desde la liberación de las responsabilidades políticas de búsqueda de bienestar personal y comunitario esenciales al ser humano. En contraste, la superación de una sociedad de únicamente electores, implica el compromiso y participación ciudadano, y ello como una alternativa surgida de la confianza y la fe innatas al hombre como un continuo "todavía-no".

Cuarto, la recuperación del papel de la memoria como uno de los grandes aportes de la religión a la política, frente a los planteamientos que abogan por una desconexión del legado del pasado, llegando incluso a establecer la posibilidad de "una cultura de la retirada, de la discontinuidad y el olvido" (p. 151). Como ya se ha expuesto, el contexto latinoamericano incluye variables relacionadas con la tradición, la familia, la tierra, que constituyen un vínculo con la historia y la cultura como expresión de la aspiración humana a la eternidad y la infinitud; en Colombia específicamente, la realidad del conflicto armado es una fuente de memoria histórica cuyo potencial aún permanece desaprovechado. Esta conexión pasado-futuro que en la modernidad tardía amenaza con una ruptura inminente, permite la ubicación en un contexto global, sin perder de perspectiva la necesidad de apostarle a una formación que explote las potencialidades de nuestra idiosincracia y les permita a las comunidades un papel de transformación social de la mano del desarrollo personal.

En conclusión, frente al "juego de la inclusión obligatoria y la exclusión forzosa" (p. 170), establecido en la sociedad contemporánea, se hacen necesarias comprensiones alternativas en lo educativo y lo político. Sin embargo, la modernidad nos ha legado una visión basada en paradigmas de "desarrollo" y "progreso" que se han convertido para América Latina en expresión de una situación insuperable donde la percepción de desventaja y atraso, impide la consideración y potenciación de las formas de conocimiento propias, que a su vez pueden generas formas de producción alternativas. 
En este sentido, el rol del sistema educativo es fundamental y de allí surge la necesidad de verificar aplicaciones en el campo escolar, que en reconocimiento de la producción científica y pedagógica que se gesta en las instituciones de básica y media, por una parte, implique conexiones de la escuela con la comunidad propia y la sociedad en general, y por otra, atienda a la trascendencia como dimensión fundamental con incidencias reales en el campo del desempeño político de los individuos así como de la producción cultural. En ese horizonte de sentido, se inscribe el proyecto La Educación Religiosa Escolar, como herramienta de formación política. Los aportes de los planteamientos expuestos, se dan en los siguientes términos.

Junto a la exposición de las raíces filosóficas y las implicaciones políticas de la Educación Religiosa como esfuerzo didáctico, se han visibilizado relaciones de la Fe como dimensión antropológica con el desempeño político, y ello en reconocimiento de la situación del ser humano en el contexto de la sociedad del riesgo. De tal forma, las ideas expuestas han contribuido a la solidez y pertinencia del problema de investigación, permitiendo una articulación de la dinámica escolar con alcances mayores a nivel social; asimismo, evidencia una tradición académica que sugiere derroteros por los cuales puede encaminarse la construcción teórica propia. Desde esa perspectiva, la necesidad de atender a las dinámicas sociales y culturales de la escuela más que a un referente teórico preestablecido, comporta una comprensión de la Educación Religiosa Escolar coherente con las circunstancias actuales, es decir, con un horizonte de sentido que establezca vínculos entre la humanidad y sus retos sociales, políticos y culturales. Ahora bien, las derivaciones prácticas de este propósito a nivel escolar, necesariamente suponen una construcción colectiva congruente con el cambio significativo en las bases epistemológicas del área; en otras palabras, que el giro epistemológico no derive únicamente de la teoría como algo ajeno a los procesos particulares de las instituciones. De esta forma, se daría pie a reflexiones religiosas y políticas sobre temas como el cuerpo, la tierra y las relaciones interpersonales, con comprensiones alternativas a los sistemas de referencia tradicionales concebidos en la modernidad. 


\section{Referenciais}

BAUMAN, Z. Cultura de Residuos. En Vidas desperdiciadas: La modernidad y sus parias, Buenos Aires: Paidós, 2004. p.123-171.

BERIAIN, J. (Comp.). Las consecuencias perversas de la modernidad: Modernidad, contingencia y Riesgo. Barcelona: Anthropos, 1996.

BEUCHOT, M. Hermenéutica Analógica y Religión. Theologica Xaveriana 60 (169), 2010. p. 25-45.

ESPINAL, C. (2006). A propósito de la universidad. Problemas, contextos y alternativas. Universitas Humanística, 62, 2006. p. 91-107.

PANNIKAR, R. Mito, Fe y Hermenéutica. Barcelona: Herder, 2007.

Recebido: $10 / 11 / 2015$

Received: $11 / 10 / 2015$

Aprovado: $02 / 12 / 2015$

Approved: 12/02/2015 